Pedagogía y Saberes No. 51

Universidad Pedagógica Nacional

Facultad de Educación. 2019. pp. 145-154

\title{
Olga Lucía Zuluaga Garcés: 40 años de historiar y conceptualizar la Pedagogía en Colombia
}

Entrevista

Olga Lucía Zuluaga Garcés: 40 Years of Historicizing and Conceptualizing Pedagogy in Colombia

Olga Lucía Zuluaga Garcés: 40 anos de historização e conceituação da Pedagogia na Colômbia

Miguel Ángel Martínez Velasco*

\section{Para citar este artículo:}

Martínez, M. (2019). Olga Lucía Zuluaga Garcés: 40 años de historizar y conceptualizar la Pedagogía en Cololmbia. Entrevista, Pedagogía y Saberes, 51, 145-155

Profesor de la Universidad del Valle, Instituto de Educación y Pedagogía. Magister en Educación de la Universidad de Antioquia (UdeA) e investigador del Grupo de Historia de la Práctica pedagógica (GHPP).

Correo electrónico: miguel.martinez.velasco@correounivalle.edu.co

Código orcid: https://orcid.org/0000-0001-9872-547X 
En los momentos que vive la Pedagogía, el trabajo histórico de orientación epistemológica debe ser parte constitutiva de sus conceptualizaciones permitiendo que la vuelta al pasado la coloque en una apertura hacia nuevas posibilidades.

Olga Lucía Zuluaga Garcés (1999a)

\section{Introducción}

Presentar a la profesora Olga Lucía Zuluaga Garcés constituye todo un desafío intelectual, por cuanto ella, como sujeto de saber (Zuluaga, 1999b) "puso en práctica un conjunto de enunciados" (p. 149) procedentes de distintos campos de saber, entre ellos la historia francesa y alemana, la epistemología de las ciencias y la filosofía; incluso fue seducida en sus inicios por el marxismo cuando estudiaba en la Universidad de Antioquia para dar cuenta de otros modos de existencia de la pedagogía. La profe Zuluaga forma parte de un movimiento de saber y de poder que posibilitó en Colombia, hacia finales de la década de 1970, la apropiación del posestructuralismo como un enfoque epistemológico que permitió problematizar la existencia de la pedagogía más allá del enrarecimiento a la que fue reducida por parte de las ciencias de la educación. Han pasado 40 años desde que aquella estudiante de la Licenciatura en Educación: Filosofía e Historia se lanzó a fundar una escuela de pensamiento cuya bandera fue la recuperación de la historicidad de la pedagogía, y desde allí, a desnaturalizar las verdades que se tejían (y se siguen tejiendo) en torno a la escuela, el maestro, la enseñanza, el método, la infancia, el alumno, el conocimiento, la didáctica, los movimientos magisteriales, la práctica pedagógica, entre otros objetos de saber.

Las contribuciones de la profesora Zuluaga a la comunidad académica, tanto nacional como internacional, son bastantes; sin embargo, hoy es posible señalar, con la madurez y la experiencia de cuatro décadas dedicadas a la investigación histórico-pedagógica, aún en medio de la crisis de la pedagogía moderna (Noguera, 2016), la consolidación de un campo de saber en el que tiene existencia la pedagogía como práctica, como saber, como disciplina, como campo conceptual.

La construcción de un campo de saber para la pedagogía en Colombia se evidencia a partir de las investigaciones que la profesora Zuluaga ha liderado a través de la apropiación de la memoria activa del saber pedagógico para llevar a cabo la reconceptualización de las prácticas educativas y pedagógicas en la contemporaneidad; la configuración de las escuelas normales, facultades e institutos de educación como instituciones productoras de este saber; la construcción del Archivo Pedagógico Colombiano (ARPE) desde la Colonia hasta la segunda mitad del siglo Xx, la configuración de una línea de estudios científicos en educación en el interior del Departamento de Ciencia, Tecnología e Innovación (Colciencias); el diseño del Instituto Nacional Superior de Pedagogía, la creación del enfoque histórico de la práctica pedagógica; la producción de más de 100 documentos entre los que se destacan artículos, libros, capítulos y ponencias en eventos tanto nacionales como internacionales, son algunas de las superficies sobre las que es posible localizar su amplia trayectoria académica para la emergencia de la pedagogía como campo de saber.

Olguita, como algunos de sus amigos más cercanos la llaman, también se ha caracterizado por su liderazgo en la creación de instituciones de saber entre las que se destacan tres: fundadora del Grupo de Investigación Interuniversitario Historia de la Práctica Pedagógica en Colombia (GHPPC), gestora del primer Doctorado Interinstitucional en Educación (DIE) en nuestro país y cogestora del Centro de Memoria Educativa y Pedagógica del Instituto para la Investigación Educativa y el Desarrollo Pedagógico (IDEP) y la Universidad Pedagógica Nacional.

Como investigadora principal, ha estado al frente de más de 16 proyectos interuniversitarios todos financiados con recursos públicos procedentes de Colciencias, del IDEP y la Universidad de Antioquia. Esta entrevista es un pretexto para conversar con ella y volver sobre algunos pasajes de su obra para seguir problematizando el presente como un ejercicio ético, político y epistemológico en torno a la crisis de la pedagogía, de la escuela, del oficio del maestro y la profesión docente. Si bien su trabajo se localiza en Colombia, sirve para que desde otras latitudes se interrogue por otras formas de existencia de la pedagogía.

Miguel Angel Martinez Velasco (MAMv). Profe Zuluaga, su pasión por la Pedagogía no es un asunto del azar. Su encuentro debió haber estado cruzado por asuntos que pasan por lo personal hasta lo académico. ¿Podría compartirnos algunos de ellos?

Olga Lucia Zuluaga Garcés (olzG). Profesor Miguel, mi encuentro con la pedagogía no inició en la universidad, se dio en el interior de mi familia. Mi vocación docente y pedagógica proviene de una amplia tradición de profesores de la cual heredé mis gustos por la literatura, en especial la poesía, al igual que las primeras preguntas que empecé hacerme en torno a la escuela y al oficio del maestro. Mi abuelo materno, Sacramento Garcés Escobar, maestro de la juventud, fue una de las personas en escribir las 
primeras genealogías familiares de Envigado. Mi padre, Arturo Zuluaga, se desempeñó como profesor de español y literatura en varios colegios de secundaria de Envigado. Mi tío paterno, Alfredo Zuluaga y Gutiérrez, fue también docente, autor de varios libros pedagógicos como Alma infantil: psicopaidología (1938) y Dramas infantiles (1957), a través de los cuales es posible ver algunas apropiaciones escolanovistas para educar a la infancia hacia la década de 1930. Emilia, Inés, Arturo y Alfredo -la familia Zuluaga Gutiérrez- eran enamorados de la docencia: ellos publicaron en Medellín, entre 1931 y 1945, el primer periódico infantil que hubo en Antioquia llamado Mi amiguito: periódico infantil y del hogar (Zuluaga y Gutiérrez, 1933). Aún conservo algunos ejemplares de ellos como parte de mis tesoros. Mi tío Hernando Garcés Uribe también influenció en mi encuentro con la poesía. De él recuerdo la publicación de un libro amplio de poemas titulado El amor junto al llanto (1948).

Sin embargo, fue mi papá quien más influenció para despertar en mí el interés y el amor por la literatura. Él quería que yo fuera escritora, pero literaria. Al concluir el bachillerato me enamoré de la filosofía y la historia, así que ingresé a la Universidad de Antioquia a estudiar estas disciplinas; cabe anotar que con los años cumplí en parte el sueño de mi padre: ser escritora... pero de textos muy académicos. Recuerdo que en mi infancia y juventud estuve rodeaba por la educación y los libros, tradición que conservo en mi biblioteca donde en diferentes estanterías conservo textos de historia, educación, pedagogía, sociología, psicología, literatura, poesía, entre otros. Mi padre era un enamorado de la docencia y cultor del idioma español, vivía rodeado de diccionarios y de grandes obras de la literatura. Él me enseñó que para escribir hay que redactar claramente y con buena ortografía.

Por otra parte, desde que empecé a estudiar la Licenciatura en Educación: Filosofía e Historia, en la Universidad de Antioquia, por allá en los años $1970 \mathrm{mi}$ interés por la pedagogía se hizo más visible especialmente a través del curso de Filosofía de la Educación (2003/1971) que enseñaba el profesor Alberto Restrepo en el Instituto de Filosofía. Allí tuve mis primeros acercamientos al pensamiento de Michel Foucault. Leímos La arqueología del saber, Las palabras y las cosas y El orden del discurso. Recuerdo que las clases de historia no me llamaban mucho la atención. Influenciada ya por la noción de archivo de Foucault, la lectura de los libros sobre historia de la educación me generaban malestar, pues la recurrencia a la legislación educativa y a los cambios que se producían según los gobiernos de turno no daban cuenta de la pedagogía sino de la educación. Allí se empezó a gestar mi trayectoria académica o, como diría Foucault, se empezaron a gestar las condiciones de existencia para el camino que empecé a transitar.

MAMv. Desde la licenciatura, usted empezó a visibilizar un problema propio de la modernidad en Occidente: la diferenciación entre la educación y la pedagogía recurriendo a la relación disciplinar filosofía e historia.

oLzG. Sí, profesor Miguel, y sin terminar aún la Licenciatura empecé a cranear un proyecto de investigación donde la filosofía y la historia tuviesen un punto de encuentro con la pedagogía con miras a ir a planteando un proyecto de largo aliento. El proyecto que presenté al Centro de Investigaciones Educativas de la Facultad de Educación, lo titulé Filosofía y pedagogía. Presentación metodológica y directrices del trabajo (1976) y dio como resultado dos folletos: Didáctica y conocimiento (1977) y Colombia: dos modelos de su práctica pedagógica durante el siglo XIX (1978). Estos primeros trabajos se constituyeron en la apropiación del primer Foucault principalmente usé La arqueología del saber (1982) para indagar las primeras formas de existencia de la pedagogía en Colombia durante el siglo XIX, y Vigilar y castigar (1981) para analizar en este mismo periodo la relación entre la enseñanza y las prácticas disciplinares a partir de la apropiación del método lancasteriano. Filosofía y pedagogía (Zuluaga, 1976) representa una aproximación conceptual, metodológica y epistemológicamente para hacer una historia de la pedagogía como saber, de allí su importancia para la realización de futuras investigaciones, muy pertinentes para seguir enfrentando su subordinación en la actualidad. Para la construcción de la noción de horizonte conceptual de la pedagogía (Zuluaga, 1999c, p. 50), leí El orden del discurso (1970), allí Foucault plantea que, para pertenecer a una disciplina, una proposición debe inscribirse en cierto horizonte teórico, en nuestro caso la pedagogía se ha constituido bajo un horizonte conceptual, el cual no se daría sin el campo conceptual y los conceptos vigentes de los pedagogos clásicos.

MAMv. Las primeras lecturas que usted hace de Foucault no pretendieron localizar a la pedagogía como una teoría sino como un saber.

olzG. Así es. Mis primeros acercamientos a $L a$ arqueología del saber (Foucault, 1982) me permitieron localizar en el saber una forma de existencia más local -menos universal como lo hace la ciencia-, más cercana para dar cuenta de la dispersión del archivo pedagógico que se empezó a configurar con el proyecto Filosofía y pedagogía (Zuluaga, 1976). Los primeros usos permitieron a los investigadores del GHPPC, en el marco del proyecto 
interuniversitario "Hacia una historia de la práctica pedagógica" (Zuluaga, 1980), reescribir la historia de la pedagogía en Colombia desde la Colonia hasta el siglo xx en registros como la producción de saber de los pedagogos y los maestros; los procesos de apropiación e institucionalización de la ciencia, las artes, las disciplinas y del saber popular para educar a través de la enseñanza, ya sea a través del Estado, las instituciones formadoras de maestros, la prensa, los manuales y enciclopedias; las transformaciones de dichos saberes por efecto del poder; los registros propios de las instituciones; las posiciones de sujetos; entre otros tipos de registros (visuales, auditivos, incluso corporales). De allí que sostengo que el saber es el espacio más plural del conocimiento, espacio que hoy gracias a la recuperación de la historicidad de la pedagogía permite establecer unos linderos epistemológicos, pero que no se cierra al trabajo con otros saberes a través de los campos conceptuales.

Nosotros empezamos a poner los ojos en aquellos archivos que para la historia clásica no representaban valor; mientras los historiadores sociales privilegiaban los archivos que provenían de las macroestructuras sociales, el GHPPC se ocupó de aquello que emergía a partir de las relaciones entre los sujetos, las instituciones y los discursos, lo que nos permitió pasar de lo macro a lo micro, es decir saltar del Estado a las luchas de saber/poder/subjetivación.

Otro elemento para destacar la elección del concepto arqueológico del saber fue su relación con la práctica. Para Foucault no existe una separación entre la teoría y la práctica, ambas forman parte de una misma unidad que funciona de manera independiente sin subordinación alguna; es decir que saber pedagógico y práctica pedagógica son las dos caras de una misma moneda.

También habría que aclarar que hacia finales de los años 1970 me incliné a pensar la pedagogía como una disciplina que emerge en un espacio plural como el saber pedagógico en aras de la sistematización del discurso de la enseñanza. Fue una apuesta política y epistemológica frente a las demandas de las historias de la ciencia que le restaban lugar al discurso pedagógico y a la instrumentalización que hizo de la pedagogía una técnica para la transmisión de los discursos forjada por las ciencias de la educación a nivel social. Hoy puede seguir siendo una opción para pensar otros modos de existencia de la pedagogía para quienes argumentan su epistemologización. Yo prefiero, desde mi experiencia en el Movimiento Pedagógico y en el acompañamiento a las escuelas normales del país, que un camino - no el camino- para seguir construyendo una comunidad académica es la vía del saber pedagógico.
MAMV. Su relato de cómo se hizo maestra universitaria da cuenta, en palabras del profesor Alberto Echeverri, de dos rostros magisteriales propios de nuestra cultura pedagógica colombiana: el que se configura en la escuela primaria, a través de la pedagogía, y el de las disciplinas, que toma fuerza en la escuela secundaria y se consolida en la universidad.

olzG. Yo nunca fui maestra ni de primaria ni de secundaria, pero sí formaba maestros en la Facultad de Educación de la Universidad de Antioquia, recién había recibido el título de Licenciada en Educación: Filosofía e Historia, en 1975. Yo llevaba en mi mente y en mi alma a los maestros, vivía pendiente de los movimientos sindicales de ellos. Inclusive, hice con el doctor Julio Puig, una compilación del Movimiento Sindical de los Maestros Colombianos titulada Movimiento de maestros en torno al Estatuto Docente 1972. Legislación, análisis y pronunciamientos (Zuluaga y Puig, 1974a) y Documentos teóricos internos al movimiento estudiantil 1971 (Zuluaga y Puig, 1974b), cuando Luis Carlos Galán era ministro de Educación. Retomando la metáfora que propone el profesor Echeverri (1998) a través de un lenguaje narrativo-literario me veo a mi misma y reconozco como propios los rostros de los maestros de los diferentes niveles de enseñanza; nunca fui indiferente a las exclusiones, a los sometimientos de la ley, al desconocimiento de su individualidad, al encierro, a las separaciones entre el saber pedagógico y los contenidos disciplinares que le impone el poder.

MAMV. A propósito de su relación con los movimientos magisteriales, ¿cuáles fueron sus aportes a la consolidación del Movimiento Pedagógico de los maestros colombianos? ¿Considera pertinente volver a ellos para enfrentar la crisis de la escuela, del maestro, de la pedagogía moderna?

oLZG. Aunque nunca busqué reconocimientos por los maestros y los líderes del Movimiento Pedagógico, siempre he luchado por los maestros y su saber. Mi estrategia fue reconocerlos a través de la escritura, principalmente a través de la Revista Educación y Cultura y de las investigaciones que dieron origen al GHPPC. Ahora bien, mi primer vínculo a las luchas de los maestros se dio en la compilación sobre el movimiento sindical de los maestros colombianos, considero que este trabajo fue desbrozando el camino para historiar los aspectos políticos, sociales, culturales e intelectuales de las luchas de los maestros, pues era necesario iniciar un archivo, para la tarea de historiar y luego conceptualizar, ya que el archivo permite ubicar lo decible y lo visible, condición para construir un campo pedagógico que permitiera a los maestros la reconceptualización de su práctica pedagógica a través de la memoria activa del saber pedagógico. 
Tanto para mí como para los miembros del GHPPC, el surgimiento del Movimiento Pedagógico es un acontecimiento de saber y poder, que en palabras del profesor Humberto Quiceno (2002) nos ubica en la recuperación de la historicidad de la Pedagogía:

¿Por qué fue pedagógico? Su nombre se debe al interés de rescatar la pedagogía. Este rescate apuntaba a volver a poner de presente la pedagogía activa que había existido en Colombia en la época liberal y a volver los ojos y el pensamiento a la pedagogía universal, a la historia de la pedagogía ya los grandes pedagogos. (p. 105).

Mi segundo vínculo a las luchas magisteriales fue plantearles a las Facultades de Educación el desafío de constituirse en instituciones productoras de saber pedagógico a partir del artículo "Las facultades de educación y el Movimiento Pedagógico" (Echeverri y Zuluaga, 1987). Allí hago un llamado a estas instituciones para que se articularan a los cambios que, desde el Movimiento Pedagógico, se estaban dando en la comunidad intelectual y en la movilización de los maestros, lo cual significaba para ellos emprender la búsqueda de una nueva individualidad sostenida por un saber propio, de tal manera, que se les distinguiera en el campo intelectual y los situara en la sociedad como trabajadores de la cultura, quienes por su accionar intelectual, cultural y social, encontraran su espacio en el saber pedagógico, ligado a las relaciones con otros saberes y disciplinas afines. Maestros que encontrasen en su práctica pedagógica una estrategia para vincular la enseñanza a la sociedad civil y un lugar de producción de su saber:

Entiéndase bien que el Movimiento Pedagógico situó la enseñanza en el terreno de la hegemonía, en donde no es posible dar solución a los problemas de la escuela, el maestro, el alumno, la práctica pedagógica y las políticas educativas, sino por medio de una concepción pedagógica que enfrente decididamente el despojo que sufre el maestro en el terreno del conocimiento, sin dejar de pensar los demás rasgos de su existencia social: su ser económico, sexual, político y lingüístico; en estos territorios indudablemente ha sido víctima de odiosos procedimientos de discriminación y exclusión. (Echeverri y Zuluaga, 1987, p. 34).

Si bien la situación de los maestros colombianos no ha cambiado de raíz, los intelectuales estamos llamados a trabajar de la mano con los maestros, apoyándolos en sus luchas y perfilando reformas que conviertan las facultades de educación en finos soportes de las búsquedas de los maestros.
MAMv. Un sello distintivo de su obra es la recurrencia a la historia para conceptualizar la pedagogía a través del análisis arqueológico de los distintos registros que constituyen hoy el archivo pedagógico colombiano. ¿Cuáles fueron los argumentos que construyó para sustentar hacer una historia de la pedagogía y no de la educación tal y como se venía haciendo por parte de los historiadores?

oLzG. Los intelectuales -aquí me refiero a los historiadores o ensayistas- se basan en las conceptualizaciones que los antecedieron en busca de discontinuidades o rupturas que ellos pueden resolver, o de nuevas conceptualizaciones producidas en su propia obra o en otros intelectuales cercanos. La historia debe esbozar planteamientos o conclusiones derivadas de los documentos que analiza y en mi caso se trataba de combinar el estudio de los pedagogos clásicos con hechos sociales y políticos, advirtiendo así que la educación no está desligada de la pedagogía, porque aquella puede encontrar en las prácticas pedagógicas formas de permanencia o de coexistencia que se articulan con un campo de presencia.

Conociendo ya que para hacer historia de los saberes según Foucault se necesitaban registros (fuentes primarias en el lenguaje de los historiadores) de diversas procedencias y que todos ellos formaban una trama de relaciones que el autor llamaba archivo y práctica discursiva, presenté con el profesor Alberto Echeverri un corto proyecto de tres meses para ir a conocer los documentos de los archivos y bibliotecas ubicados en Bogotá.

MAMV. ¿De dónde vino la idea de fundar el Grupo Historia de la Práctica Pedagógica en Colombia?

olzG. El Grupo Historia de la Práctica Pedagógica en Colombia se podría decir que es un acontecimiento de saber, me perdonas que sea tan foucaultiana (risas). ¡Lo que pasa es que yo quiero mucho a mi calvo! No fue algo pensando, no obedeció a una política institucional o del Estado a través de Colciencias, surgió del azar, del encuentro pasional que se empezó a tejer con el archivo cuando era auxiliar de investigación en el CEDED de la Facultad de Educación de la Universidad de Antioquia. Esa pasión por la historia, por la pedagogía, por el maestro, por la escuela, por la infancia, llevó a que un grupo de profesores universitarios formados en plena efervescencia de las ciencias de la educación de origen norteamericano asumieran el desafío de investigar un objeto tan raro como la pedagogía a través de la arqueología del saber.

Recuerdo que hicimos un pacto: Stella Restrepo, de la Universidad Nacional de Colombia, sede Bogotá, se encargaría de investigar a los jesuitas; el profesor 
Alberto Martínez Boom, de la Universidad Pedagógica Nacional, de estudiar la Colonia, y el profesor Alberto Echeverri y yo, de la Universidad de Antioquia, nos encargamos del siglo XIX. El profesor Humberto Quiceno, de la Universidad del Valle, trabajaba la historia de principios del siglo xx. Como auxiliar de investigación se fue integrando el profesor Óscar Saldarriaga, en calidad de estudiante de Historia de la Universidad de Antioquia. Durante el Movimiento Pedagógico se fueron vinculando los docentes Alejandro Álvarez, quien venía de trabajar directamente con el sindicato de Fecode; Javier Sáenz, a quien conocimos a través de su trabajo con la Fundación Foro, y Carlos Ernesto Noguera, de la Universidad Pedagógica Nacional. No puedo pasar por alto los aportes del profesor Vladimir Zapata como docente de la Universidad de Antioquia.

En el marco de la reforma a las escuelas normales, hacia 1997, el grupo crece exponencialmente: se vincula la profesora Dora Marín, quien venía de la Escuela Normal Superior La Paz, de Bogotá; como estudiantes de doctorado se integran los profesores Rafael Ríos, Bernardo Barragán y Arley Ossa. Desde la Universidad del Cauca nos estuvo acompañando la profesora Elizabeth Castillo. El docente Javier Fayad lo hace desde la Universidad del Valle. Hoy somos un grupo conformado aproximadamente por 30 investigadores entre profesores, estudiantes de pregrado y posgrado. Quiero resaltar la experiencia de formación llevada a cabo por el profesor Alberto Echeverri quien desde finales de la década de 1990 creyó en la formación y en la investigación en el pregrado y cuyo resultado se puede ver en la conformación del grupo en Medellín, sin dicho esfuerzo humano e intelectual hoy no tendríamos a quién heredarle la tradición de 40 años. Junto a él, la profesora Rosa María Bolívar ha impulsado la investigación a través de los semilleros de investigación en el país, especialmente a través del Semillero de Investigación en Pedagogía (SIP). Junto a ella se destaca la experiencia de Rafael Reyes y Óscar Saldarriaga, de la Pontificia Universidad Javeriana, de Bogotá, con el semillero "El oficio del maestro" y la llevada a cabo por los profesores Carlos Noguera y Dora Marín con su grupo de estudio en la Universidad Pedagógica Nacional. No somos una iglesia, ni una secta pedagógica, somos un grupo de personas inquietas, inconformes y rebeldes por el lugar que se le ha conferido y se le sigue asignado a la pedagogía.

MAMV. Un sector de los historiadores tanto colombianos como extranjeros ven como extraño el relato histórico que usted ha venido produciendo desde hace 40 años, de recurrir a la historiografía francesa y alemana para llevar a cabo la historicidad de la pedagogía.
oLzG. Profesor Miguel, usted pone sobre la mesa un tema bastante sensible: las tensiones entre historiadores y pedagogos. Desde mi paso por la Facultad de Educación de la Universidad de Antioquia empecé a vivir en carne propia las luchas entre estos dos campos de saber provenientes de las ciencias de la educación. Lo primero que tendría que aclarar es que me reconozco como historiadora de la pedagogía y no como historiadora de las ideas pedagógicas, de allí que no recurro a la historia desde el pasado para pensar el presente, tal y como lo hacen dichos historiadores; sino que parto de la problematización del presente para encontrar un punto de partida donde lo político, lo social y lo pedagógico formen una trama susceptible de ser historiada. El discurso oficial también forma parte de dicha relación, solo que no determina las condiciones de emergencia de la pedagogía en sus múltiples formas de existencia.

Mi trabajo intelectual siempre tuvo como propósito, como usted lo señala en su pregunta, la historicidad de la pedagogía, mas no la historia de la pedagogía. Las tensiones que experimentan los historiadores también las vivimos los pedagogos cuando somos interrogados desde la exterioridad. Mi investigación doctoral (Zuluaga, 2000) es un ejemplo o, si se quiere interpretar, una muestra de que la historiografía de la educación y la historicidad de la pedagogía pueden encontrarse y relacionarse. Otro ejemplo más desde el orden colectivo fue la investigación de historia comparada (Zuluaga et al., 2004a, 2004b), en la que confluyeron historiadores iberoamericanos con una amplia trayectoria académica como Gabriela Ossenbach, Luz Elena Galván, Agustín Escolano, entre muchos otros. Paul Veyne (1984) afirma:

La historia no es más que lo que hacemos de ella; no ha dejado de cambiar, pues su horizonte no es eterno. Lo que hace Foucault se llamará historia -y por ende lo será-, si los historiadores aceptan el regalo que se les hace sin rechazarlo por inalcanzable. (p. 238).

Parafraseando a Veyne, el relato histórico que he venido construyendo de la mano con el GHPPC forma parte de un esfuerzo intelectual que hace ver en la historia esa otra dimensión que la caja de herramientas de Foucault permitió develar: la historicidad de los saberes más allá de las estructuras y de las prácticas sociales a partir de las relaciones que se tejen entre discursos, instituciones y sujetos (IDS).

De otro lado, he recibido muchos cuestionamientos provenientes de los historiadores sociales, quienes, al no diferenciar epistemológicamente la educación de la pedagogía, reducen la complejidad de lo que acontece en torno a la formación del sujeto a partir 
de las múltiples relaciones que establece la enseñanza con la cultura, la política, la ética. La influencia de la sociología (Bernstein y Díaz, 1985) sigue situando a la pedagogía como un mecanismo de reproducción del Estado, de allí que

[...] los énfasis exclusivamente sociales para historiar la educación opacan la posibilidad de hacer historias de prácticas específicas, relacionadas con instancias sociales y políticas, pero delimitadas. Al mismo tiempo, sobreestiman el papel de la relación educación educación-sociedad [...]. Lo que en nuestro medio se llama historia social de la educación no agota todo lo que a propósito de la educación y la pedagogía puede ser objeto de historia [...], la educación no está desligada de la pedagogía [...], porque aquella encuentra en las prácticas pedagógicas formas de permanencia o de cambios. (Zuluaga y Martínez, 1996, pp. 56-57).

También tendría que aclarar que el enfoque histórico de la práctica pedagógica no se lleva a cabo al margen de las prácticas sociales, parte de ellas; solo que las condiciones de existencia para que emerja un saber, en este caso la pedagogía, no están determinadas por ellas sino por un entramado de saber/poder/ subjetivación. Como diría el poeta español Ramón de Campoamor (1892):

$\mathrm{Y}$ es que en mundo traidor

Nada es verdad ni es mentira:

Todo se ve del color del cristal con que se mira. (p. 183).

MAMV. ¿Cómo ve las críticas que han mirado como extrañas, las referencias históricas que hace, sobre todo a los historiadores franceses, en particular, a Michel Foucault?

oLzG. Profesor Miguel, entiendo que ello no tiene nada de extraño. Los intelectuales e historiadores se citan unos a otros, ya sea para apoyarse o para cuestionar. Basta con mirar la bibliografía de sus escritos para deducirlo. Michel Foucault refiriéndose a sus críticos argumentaba: “No me pregunten quién soy, ni me pidan que permanezca invariable: es una moral de estado civil la que rige nuestra documentación. Que nos dejen tranquilos cuando se trata de escribir" (Foucault, 1979, p. 29). Mi trabajo en analogía a lo expresado por Foucault no existe y no se construye al margen de otros textos, sino que está cruzado por diferentes autores quienes representan a su vez tradiciones:

Por más que el libro se dé como un objeto que se tiene bajo la mano, por más que se abarquille en ese pequeño paralelepídico que lo encierra, su unidad es variable y relativa. No bien se le interroga pierde su evidencia; no se indica a sí mismo, no se construye sino a partir de un campo complejo de discursos. (Foucault, 1979, p. 37).

Volviendo a lo extraño que ven en mi trabajo algunos historiadores, especialmente la influencia de Michel Foucault (2010) en mi obra, ellos no comprenden que unos autores necesitan de la obra de sus maestros o de sus compañeros de investigación. Es decir, quien escribe se inscribe en un grupo de intelectuales cercanos, así es como se forman las escuelas de pensamiento sobre cualquier tema de investigación, por ejemplo, la escuela de los Annales en Historia y los grupos que se forman alrededor de un "fundador de discursividad" (p. 20), como es el caso de Freud y sus discípulos u opositores, permaneciendo en un mismo campo, así se extiendan en un abanico de corrientes.

Mamv. Otro de los sellos que distinguen su obra es la recurrencia a los pedagogos clásicos para problematizar el presente. ¿Cuál es la vigencia de pedagogos como Comenio, Rousseau, Herbart y Pestalozzi, para seguir pensando los procesos de formación de maestros?

oLzG. Para responderle esta pregunta recurro primero a la noción saber como un movimiento en un campo que así no sea inmediato, en términos del tiempo, puede haber conservado planteamientos, preguntas, percepciones $\mathrm{u}$ horizontes que han abierto el paso a conceptualizaciones y preguntas que permiten construir una memoria que merece llamarse memoria activa del saber pedagógico. Esta nos remite a los pedagogos clásicos tanto con un sentido crítico como constructivo para problematizar el presente, como enseñanza, formación, instrucción, educación, preceptor, maestro, infancia, experiencia, individualidad, y desde dicha memoria probablemente tejer el presente. Creo que este debe ser un curso o un seminario primordial en la formación de maestros en la actualidad, independiente del nivel de enseñanza en el que ejerza.

Considero que "el desprecio por la historia es hijo de una estéril actitud que puede resumirse en la frase 'tierra de nadie'" (Zuluaga y Echeverri, 1990, p. 185). 0 sea, es preciso mirar el pasado para entender el presente, y ubicar en el camino zanjas, rupturas, desvíos, discontinuidades, áreas comparables, fases, etc. Por lo anterior, es necesario estudiar lo que llamo pedagogos clásicos, quienes han elaborado conceptos, métodos y problematizaciones que, aunque fueron vigentes, en su época, se cruzan con nuestro presente, de una u otra manera, y cobran presencia en la actualidad. "Así como las ciencias tienen su pasado actual, mediante la historia recurrente; las disciplinas, en cambio, lo deben hacer mediante las problematizaciones actuales, cuya solución se requiere para continuar en búsqueda de 
sistematicidad" (Zuluaga y Echeverri, 1990, p. 87). En nuestra cultura latinoamericana, la pedagogía como saber pedagógico tiene lugar en la contemporaneidad recurriendo a la noción de memoria activa del saber pedagógico (Zuluaga y Marín, 2006; Zuluaga y Herrera, 2006; Zuluaga, 2016).

No pretendo declarar como eternos los pedagogos clásicos, sino que sus planteamientos en su transitar por diferentes sociedades y culturas se mueven y pueden cambiar en sus conceptos y métodos, de este modo se inscriben en el saber pedagógico. Cada pedagogo clásico y criollo, como usted llama a los propios, aporta insumos importantes para enfrentar la crisis educativa que hoy estamos viviendo. Para volver a los pedagogos, es necesario situarlos en el horizonte conceptual de la pedagogía. Creo importante volver a Comenio para retomar la relación didáctica/pensamiento/lenguaje clave para enfrentar la enseñanza de los saberes escolares; de Rousseau, es importante incorporar el concepto de libertad para enfrentar la crisis educativa que hoy nos asiste, pues dota a los maestros de herramientas epistemológicas que les permiten liberarse de la carga opresora que hoy llevan ante la dificultad de educar en la contemporaneidad. Pestalozzi es vigente para pensar la educación infantil a través de los conceptos de intuición y amor maternal; de Herbart, es importante la relación entre la enseñanza, la educación y el interés como tríada que individualiza la pedagogía de otros saberes.

Es muy importante para los desarrollos actuales de la pedagogía trabajar en grupos, pues se puede ser un buen investigador "únicamente si se está preparado para trabajar con muchas teorías diferentes, y no con un solo punto de vista y experiencia" (Feyerabend, 1974, p. 31). De allí la importancia de reconocer la pedagogía como saber, por cuanto permite recorrer distintos campos de saber a través de las múltiples relaciones que se pueden tejer entre lenguaje, pensamiento, intuición, amor maternal, enseñanza, educación, interés, entre otros conceptos.

MAMV. ¿Cuáles son los problemas educativos que usted hoy requieren ser problematizados recurriendo a la historicidad de la pedagogía?

oLzG. Si bien desde hace varios años no he vuelto a participar públicamente de los debates educativos y pedagógicos, ello no significa que no esté atenta a las discusiones que se dan principalmente a través de los medios de comunicación. Intento mantenerme al día sobre lo que pasa a través de las conversaciones que mantengo con mis grandes amigos de la pedagogía: Alberto Echeverri, Stella Restrepo, Alberto Martínez Boom, Humberto Quiceno, Rafael Ríos, Dora Marín,
Carlos Noguera, Alejandro Álvarez, Martha Cerquera, entre otros tantos. Me he valido del Facebook y el WhatsApp para intentar mantenerme al día.

Hoy considero que la noción de práctica pedagógica que elaboré a partir de la noción de práctica discursiva (Zuluaga, 1999d, p. 35) para pensar relacionalmente el acontecimiento de saber-poder que teje entre instituciones, sujetos y discursos, hoy puede ser susceptible de ser apropiada no solo para seguir haciendo historia de la práctica pedagógica, sino también para pensar el campo aplicado de la pedagogía. Es una deuda que hoy tenemos con los maestros. Un ejemplo de ello es el que se dio en la Universidad Francisco de Paula Santander, sede Cúcuta; allí, en medio de las discusiones que suscitó el Decreto 0272 del 11 de febrero de 1998, se apropió la noción de práctica pedagógica para pensar la formación de maestros. De allí nació una especialización y hoy, después de muchos años de arduo trabajo, se ha venido consolidando una comunidad académica entorno a la maestría en práctica pedagógica. Por ello, me lanzo a pensar otro modo de existencia de esta noción que intenta recoger la tradición a la luz de las demandas del presente. Provisionalmente, considero la práctica pedagógica como noción metodológica que designa:

1. Las relaciones entre saberes, sujetos e instituciones.

2. Los dispositivos pedagógicos tanto teóricos como prácticos utilizados en los niveles de enseñanza infantil, primaria y secundaria.

3. Una pluralidad de nociones y conceptos pertenecientes a diversos campos de saber para educar, enseñar y formar a través de la memoria activa del saber pedagógico.

4. Las formas de funcionamiento de los discursos en las instituciones educativas donde se realizan las prácticas pedagógicas.

5. El entramado de tensiones y relaciones de saber y de poder para llevar a cabo la producción de subjetividades del maestro y del alumno.

6. Las características sociales adquiridas por la práctica pedagógica a partir de las relaciones que establece con la sociedad, la cultura y los campos conceptuales de la pedagogía.

7. Las posiciones de sujeto que demandan al maestro las distintas tradiciones pedagógicas.

8. El lugar donde el maestro produce saber pedagógico a partir de la relación entre la experiencia, la cultura y los campos conceptuales de la pedagogía. 
MAMV. Ante los cambios ocurridos en el lapso de 40 años, ¿qué tareas son primordiales para usted?

oLzG. Aunque las luchas de hace 40 años hoy no son las mismas -como fruto de la experienciadebemos seguir acompañando a los maestros en sus luchas por una mejor calidad de vida: saber, salud, prestaciones sociales, capacitación, como señalan Martínez, Unda y Mejía (2002): “De lucha por el saber y de lucha para saberse en su autonomía que libere al maestro de las ataduras que lo han convertido en sujeto de controles y dependencia" que permita hacer del movimiento pedagógico en la contemporaneidad "un movimiento intelectual" (p. 77). Hoy nos enfrentamos a pensar una escuela que ya no se ocupa de transmitir únicamente la cultura, de un maestro que se ve enfrentado a despojarse de la enseñanza como su objeto de saber, unas infancias y unas adolescencias que se resisten a ser educados -gobernados- por el adulto. En la actualidad, planteo reelaborar algunos conceptos y dedicarlos a conversar con las coyunturas del presente, y ponerlos a consideración de quienes tienen una mirada diferente. Actualmente, me ocupo de reelaborar dichas instancias (ISD), a la luz de nuevas conceptualizaciones, planteadas por investigadores del GHPPC, y de investigadores afines con el fin de conceptualizar y enfrentar las dificultades del presente. Un ejemplo de dichas reelaboraciones se sitúa en los usos de la pedagogía moderna para enfrentar la crisis de la educación de las infancias que estoy trabajando actualmente con algunos de los miembros del Grupo de la Universidad del Valle. Considero que existe una inmensa responsabilidad en las facultades de Educación que debían formar docentes con aptitudes de articularse con grupos de intelectuales en busca de una escuela capaz de instalar redes entre la formación, la enseñanza, el conocimiento, la cultura y la sociedad, recurriendo a las tradiciones pedagógicas.

\section{Referencias}

Bernstein, B. y Díaz, M. (1985). Hacia una teoría del discurso pedagógico. Revista Colombiana de Educación, 15, 105-152.

De Campoamor, R. (1892). Las dos linternas. En Doloras (pp. 183-185). París: Editorial de Garnier Hermanos.

Echeverri, A. (enero de 1998). Mi encuentro con el rostro magisterial. En III Encuentro de Investigación Educativa y Desarrollo Pedagógico en el Distrito Capital (pp. 135-148). Instituto para la Investigación Educativa y el Desarrollo Pedagógico, Bogotá.
Echeverri, A. y Zuluaga, O. (1987). Las facultades de educación y el Movimiento Pedagógico. Revista Foro, 3, 33-40.

Feyerabend, P. (1974). Introducción. En Contra el método. Esquema de una teoría anarquista del conocimiento (pp. 1-95). Barcelona: Ariel.

Foucault, M. (1970). El orden del discurso. Barcelona: Tusquets.

Foucault, M. (1981). Vigilar y castigar: nacimiento de la prisión. Buenos Aires: Siglo del Hombre Editores.

Foucault, M. (1979). Las regularidades discursivas. En La arqueología del saber (pp. 31-127). 6a. ed. Buenos Aires: Siglo del Hombre Editores.

Foucault, M. (2010). ¿Qué es un autor? Córdoba: Ediciones Literales.

Garcés, H. (1948). El amor junto al llanto. Medellín: Tipografía Bedout.

Martínez, A., Unda, P. y Mejía, M. (2002). El itinerario del maestro: de portador a productor de saber pedagógico. En H. Suárez (comps.), 20 años 1982-2002 del Movimiento Pedagógico: entre mitos y realidades (pp. 61-94). Bogotá: Cooperativa Editorial Magisterio.

Noguera, C. (2016). Una perspectiva política, ética y estética para un nuevo gesto pedagógico. En A. Martínez, A. Ruíz y G. Vargas (comps.), Epistemología de la pedagogía (pp. 103-133). Bogotá: Universidad Pedagógica Nacional, Doctorado Interinstitucional en Educación.

Quiceno, H. (2002). Movimiento pedagógico: posición crítica y lugar de liberación. En H. Suárez (comp.), Veinte años del movimiento pedagógico. 1982-2002. Entre mitos y realidades (pp. 95-126). Bogotá: Cooperativa Editorial Magisterio.

Restrepo, A. (2003/1971). Programa de curso. Revista Educación y Pedagogía, 37, 285-289.

Veyne, P. (1984). Como se escribe la Historia. Foucault revoluciona la Historia. Madrid: Alianza.

Zuluaga, O. (1976). Filosofía y pedagogía: presentación metodológica y directrices del trabajo. Medellín: Editorial Universidad de Antioquia.

Zuluaga, O. (1977). Didáctica y conocimiento. Medellín: Centro de Investigaciones Educativas, Universidad de Antioquia.

Zuluaga, 0. (1978). Colombia: dos modelos de su práctica pedagógica durante el siglo XIX. Medellín: Editorial Universidad de Antioquia. 
Zuluaga, 0. (1980). Hacia una historia de la práctica pedagógica en Colombia. Proyecto de investigación interuniversitario. Medellín: Universidad de Antioquia, Universidad Pedagógica Nacional, Universidad Nacional de Colombia, Universidad del Valle.

Zuluaga, O. (1999a). El trabajo histórico y la recuperación de la práctica pedagógica (Notas metodológicas). En Pedagogía e Historia. La historicidad de la pedagogía. La enseñanza, un objeto de saber (pp. 9-24). Bogotá: Editorial Universidad de Antioquia, Anthropos, Siglo del Hombre Editores.

Zuluaga, O. (1999b). Vocabulario metodológico. En Pedagogía e Historia. La historicidad de la pedagogía. La enseñanza, un objeto de saber (pp. 137-150). Bogotá: Editorial Universidad de Antioquia, Anthropos, Siglo del Hombre Editores.

Zuluaga, O. (1999c). Pedagogía, práctica pedagógica y sujetos de la enseñanza. En Pedagogía e Historia. La historicidad de la pedagogía. La enseñanza, un objeto de saber (pp. 44-54). Bogotá: Editorial Universidad de Antioquia, Anthropos, Siglo del Hombre Editores.

Zuluaga, O. (1999d). El saber pedagógico y su archivo. En Pedagogía e Historia. La historicidad de la pedagogía. La enseñanza, un objeto de saber (pp. 25-43). Bogotá: Editorial Universidad de Antioquia, Anthropos, Siglo del Hombre Editores.

Zuluaga, O. (2000). La educación pública en Colombia 18451875: libertad de enseñanza y adopción de Pestalozzi en Bogotá. Bogotá: Instituto para la Investigación Educativa y el Desarrollo Pedagógico, Universidad de Antioquia.

Zuluaga, O. (2016). Saberes, paradigmas y campos conceptuales. En A. Martínez, A. Ruíz y G. Vargas (comps.), Epistemología de la pedagogía (pp. 69-85). Bogotá: Universidad Pedagógica Nacional, Doctorado Interinstitucional en Educación.

Zuluaga, O. y Echeverri, A. (1990). El florecimiento de las investigaciones pedagógicas. En M. Díaz y J. Muñoz (eds.), Pedagogía, discurso y poder (pp. 175-201). Bogotá: Corprodic.

Zuluaga, O. y Gutiérrez, A. (1933). Mi amiguito: periódico infantil y del hogar. Medellín.
Zuluaga, O. y Gutiérrez, A. (1938). Alma infantil: psicopaidología. Medellín: Creset.

Zuluaga, O. y Gutiérrez, A. (1957). Dramas infantiles. Medellín: Editorial Publicaciones Pedagogía y Didáctica.

Zuluaga, O. y Herrera, S. (2006). Relaciones entre el saber pedagógico, práctica pedagógica y memoria activa del saber pedagógico. En H. Quiceno (comp.), Territorios pedagógicos: espacios, saberes y sujetos (Vol. 1, pp. 91-102). Bogotá: Universidad Pedagógica Nacional, Instituto Nacional Superior de Pedagogía.

Zuluaga, O. y Marín, D. (2006). Memoria colectiva. Memoria activa del saber pedagógico. Educación y Ciudad, 10, 59-87.

Zuluaga, O. y Martínez, A. (1996). Historia de la educación y la pedagogía: desplazamientos y planteamientos. En: A. Martínez y M. Narodowski (comps.), Escuela, historia y poder: miradas desde América Latina (pp. 55-75). Buenos Aires: Novedades Educativas.

Zuluaga, O. y Puig, J. (1974a). Documentos teóricos internos al movimiento estudiantil 1971. Medellín: Editorial Universidad de Antioquia.

Zuluaga, O. y Puig, J. (1974b). Movimiento de maestros en torno al Estatuto Docente 1972. Legislación, análisis y pronunciamientos. Medellín: Editorial Universidad de Antioquia.

Zuluaga, O. Ossenbach, G., Chagas, M, Echeverri, A., Escolano, A., Galván, L., Guyot, V., Pereira, J., Marincevic, J., Osorio, D., Paladines, C., Saldarriaga, Ó., Spedo, M., Vitareli, M., Warde, M., Zapata, V., Paladines, C. (2004a). Génesis y desarrollo de los sistemas educativos iberoamericanos siglo XIX. Bogotá: Colciencias, Universidad de Antioquia, Universidad del Valle, Universidad Pedagógica Nacional, Universidad de los Andes.

Zuluaga, O., Ossenbach, G., Narodowski, M., Quiceno, H., Sáenz, J., Vahos, A., Paladines, C., Celada, P., Lazarín, F., Álvarez, A. (2004b). Modernización de los sistemas educativos iberoamericanos siglo XIX. Bogotá: Colciencias, Universidad de Antioquia, Universidad del Valle, Universidad Pedagógica Nacional, Universidad de los Andes. 